\title{
Caracterização filogenética de amostras do vírus da imunodeficiência felina (FIV) do Estado de São Paulo ${ }^{1}$
}

\begin{abstract}
Valéria M. Lara² ${ }^{2}$ Sueli A. Taniwaki ${ }^{3}$ e João P. Araújo $\mathrm{Jr}^{3^{*}}$
ABSTRACT.- Lara V.M., Sueli Akemi Taniwaki S.A. \& João Pessoa Araújo Jr J.P. 2007. [Phylogenetic characterization of feline immunodeficiency virus (FIV) isolates from the state of São Paulo.] Caracterização filogenética de amostras do vírus da imunodeficiência felina (FIV) do Estado de São Paulo. Pesquisa Veterinária Brasileira 27(11):467-470. Departamento de Microbiologia e Imunologia, Instituto de Biociências, Universidade Estadual Paulista, Botucatu, São Paulo 18618-000, Brazil. E-mail: ipessoa@ibb.unesp.br

Feline immunodeficiency virus (FIV) is a lentivirus associated with immunologic disorders in domestic cats. Due to the high genetic variability of FIV, five subtypes (A to E) have been identified and diversity within each subtype is also frequent. The study of the genetic diversity can aid the understanding the pathogenesis and epidemiology of the disease. Therefore, the present work aimed to analyze phylogenetically FIV isolates of domestic cats from the state of São Paulo, Brazil. The sequencing of 658 bp of the gag gene from 23 samples was performed and the results were analyzed using the Tamura-Nei nucleotidic substitution method. The phylogenetic analysis showed that all viruses belong to subtype $B$, and clearly three subgroups were present within this subtype. Additionally, these results suggest a common ancestor between the FIV strains derived from Japan and one Brazilian virus. In conclusion, this work presents the first information about the genetic diversity of FIV in the state of São Paulo. Additional studies are necessary to characterize the real scenario of the distribution of FIV subtypes in the population of Brazilian cats.
\end{abstract}

INDEX TERMS: Cat, subtype, FIV, phylogeny, genotyping.

RESUMO.- O vírus da imunodeficiência felina (FIV) é um lentivírus que causa distúrbios imunológicos em gatos domésticos. Devido à alta variabilidade genética do FIV, já foram identificados cinco subtipos (A a E) e a diversidade dentro de cada subtipo é frequiente e o seu estudo pode auxiliar no conhecimento da patogenia e epidemiologia da doença. Assim, o presente trabalho objetivou analisar filogeneticamente cepas do FIV de gatos domésticos oriundos do estado de São Paulo. Para tanto, foi realizado o seqüienciamento de 658 pares de bases do gene gag de amostras coletadas de 23 animais, cujos resultados foram analisados pelo método de substituição nucleotídica Tamura-Nei. A análise filogenética

\footnotetext{
${ }^{1}$ Recebido em 5 de julho de 2007.

Aceito para publicação em 13 de novembro de 2007.

2 Departamento de Microbiologia e Parasitologia, Centro de Ciências da Saúde, Universidade Federal de Santa Maria, Av. Roraima 1000, Cidade Universitária, Bairro Camobi, Santa Maria, RS 97105-900, Brasil.

${ }^{3}$ Departamento de Microbiologia e Imunologia, Instituto de Biociências, Universidade Estadual Paulista, Botucatu, SP18618-000, Brasil. "Autor para correspondência: jpessoa@ibb.unesp.br
}

demonstrou que todas as amostras pertenciam ao subtipo B e, claramente, três subgrupos foram formados dentro deste subtipo. Adicionalmente, o resultado obtido sugeriu um ancestral comum entre as cepas do FIV oriundas do Japão e uma amostra brasileira obtida neste estudo. Em conclusão, este trabalho traz as primeiras informações sobre a diversidade genética do FIV no Estado de São Paulo. Estudos adicionais são necessários para melhor entender o cenário real e a distribuição dos tipos e subtipos do FIV na população de gatos domésticos do país.

TERMOS DE INDEXAÇÃO: Gato, subtipo, FIV, filogenia, genotipagem.

\section{INTRODUÇÃO}

O vírus da imunodeficiência felina (FIV) é um retrovírus classificado na subfamília Lentivirinae, identificado em 1986 (Pedersen et al. 1987) e responsável por distúrbios imunológicos em gatos domésticos semelhantes aos observados em pacientes infectados com o vírus da imunodeficiência humana (HIV). A situação epidemiológica mundial mostra uma ampla distribuição do FIV, com taxas de prevalência variando 
desde 2,5\% até 44\% em diferentes regiões (Hohdatsu et al. 1998, Luria et al. 2004, Bandechi et al. 2006, Levy et al. 2006, Arjona et al. 2007). No Brasil, as taxas de prevalência reportadas variam entre $3,7 \%$ e $37,5 \%$, sendo que os menores valores foram relatados em gatos assintomáticos, e os maiores em animais doentes (Reche Jr et al. 1997, Caldas et al. 2000, Lara et al. 2001, Souza et al. 2002).

Estudos prévios baseados na diversidade genética da região V3-V5 do gene env demonstraram a ocorrência de cinco subtipos diferentes, designados de A, B, C, D e E. Subseqüentemente, estudos mostraram ser possível realizar a mesma classificação genética para o FIV utilizando seqüência de nucleotídeos da região p17-p24 do gene gag (Kakinuma et al. 1995, Hohdatsu et al. 1998, Steinrigl \& Klein 2003, Duarte \& Tavares 2005). Adicionalmente, o gene gag apresenta uma taxa de conservação mais elevada que o gene env. Entretanto, as mutações encontradas fazem deste gene um candidato adequado para estudos filogenéticos (Clavel et al. 1986, Fisher et al. 1988, Greene et al. 1993, Hohdatsu et al. 1998).

O subtipo A inclui as cepas de FIV isoladas na Califórnia, Austrália e Europa (Greene et al. 1993, Bachmann et al. 1997, Pistello et al. 1997); o subtipo B abrange as cepas isoladas no Japão, na região central e leste dos EUA e Europa (Bachmann et al. 1997, Nishimura et al. 1998, Duarte et al. 2002); o subtipo C inclui as cepas isoladas no Canadá, Europa e Formosa (Sodora et al. 1994, Kakinuma et al. 1995, Inada et al. 1997); o subtipo D foi, até o presente momento, somente identificado no Japão (Kakinuma et al 1995) e o subtipo E foi identificado na Argentina e Japão (Sodora et al 1994, Pecoraro et al. 1996). Os subtipos A e B têm sido os mais freqüentemente identificados (Duarte \& Tavares 2005) e estudos recentes demonstraram a ocorrência de subgrupos dentro do subtipo B (Steinrigl \& Klein 2003, Weaver et al. 2004, Duarte \& Tavares 2005), refletindo a diversidade genética observada nos diferentes subtipos do FIV (Sodora et al. 1994, Bachmann et al. 1997).

No Brasil, pouco se conhece sobre a diversidade genética do FIV. Até o momento, foi realizado, no Estado de Minas Gerais, um estudo filogenético com 10 amostras do FIV, que identificou somente o subtipo B (Caxito et al. 2006).

$\mathrm{O}$ presente estudo objetivou analisar filogeneticamente cepas do FIV identificadas em gatos domésticos oriundos de diferentes cidades do estado de São Paulo, Brasil.

\section{MATERIAL E MÉTODOS}

Para este estudo, foram utilizadas 23 amostras do FIV detectadas pela nested-PCR (Hohdatsu et al. 1998), com pequenas modificações, de amostras de sangue de gatos domésticos oriundos de diferentes cidades do estado de São Paulo. Posteriormente, as amostras foram submetidas ao seqüenciamento da região p17-p24 do gene gag. No Quadro 1 são apresentadas algumas características dos animais, como sexo, idade, condição fisiológica, procedência e o número de acesso no GenBank da sequiência do gene gag obtida.

A extração do DNA proviral foi realizada com o kit GFX Genomic Blood DNA Purification ${ }^{\circledR}($ Amersham Pharmacia Biotech), de acordo com o protocolo do fabricante. Para amplificação do fragmento de nucleotídeos a ser sequienciado, foram desenhados dois pares de primers diferentes dos utilizados na nested-PCR, os quais amplificaram uma sequiência de nucleotídeos de 658 pares de base $(\mathrm{pb})$ da região p17-p24 do gene gag, sendo estes: FIV PCR A2 (5' ACT TCT TGG CAG GCC CTC AG 3') e FIV PCR S2 (5' AAG GAC CTC CAC AGG CTT ATC C 3'). Esses primers foram escolhidos após comparações com 10 sequiências do FIV disponíveis no GenBank através do programa OMIGA v.3.0 (Oxford Molecular Company), pois demonstraram amplificar qualquer um dos cincos subtipos do FIV e melhoram as condições da reação. A reação da PCR foi realizada em um volume final de $25 \mu$ l contendo 1 unidade de Tth DNA Polimerase (biotools ${ }^{\circledR}$ ), $2.5 \mu$ l de tampão de PCR 10X (biotools ${ }^{\circledR}$ ), $1 \mu \mathrm{l}$ de cada primer a $10 \mathrm{pmol} / \mu \mathrm{l}$ e $5 \mu \mathrm{l}$ do DNA extraído. As condições utilizadas para amplificação foram: $96^{\circ} \mathrm{C}$ por $4 \mathrm{~min}$, seguido de 30 ciclos de desnaturação $\left(94^{\circ} \mathrm{C}, 1 \mathrm{~min}\right)$, anelamento $\left(58^{\circ} \mathrm{C}\right.$, $1 \mathrm{~min})$ e extensão $\left(72^{\circ} \mathrm{C}, 1 \mathrm{~min}\right)$. Ao final da reação, os produtos foram analisados em gel de agarose a $1,5 \%$, corado com brometo de etídio $(0,5 \mathrm{mg} / \mathrm{ml})$ e comparados com marcadores de DNA de $100 \mathrm{pb}$ (GibcoBRL $\AA)$, sendo considerados positivos aqueles com tamanho aproximado de $658 \mathrm{pb}$. Como controle positivo, foi utilizada uma amostra extraída de um gato com resultado positivo através de um kit de ELISA comercial (Snap ${ }^{\circledR}$ FIV/FeLV Combo Test, IDEXX) para FIV; e como controle negativo, utilizou-se água ultrapura estéril.

Os fragmentos de DNA obtidos da PCR foram purificados diretamente ou extraídos do gel, caso apresentassem bandas espúrias, utilizando o kit GFX PCR DNA and Gel Band Purification Kit (Amersham Biosciences Inc.) de acordo com as especificações do fabricante. Em seguida o produto foi quantificado em gel de agarose comparativamente com o marcador de massa molecular (Low DNA Mass Ladder- Invitrogen). O seqüenciamento dos produtos de amplificação foi realizado em ambas as direções com os primers utilizados na PCR usando o kit DYEnamic ${ }^{\circledR}$ ET Terminator Cycle Sequencing (Amersham Biosciences) em um sequienciador automático modelo ABI Prism ${ }^{\circledR} 377$ DNA Sequencers (Applied Biosystems), seguindo as recomendações do fabricante. Cada reação de seqüenciamento foi repetida três vezes e as seqüências foram analisadas pelo programa Phred (Ewing et al. 1998) sendo aceitas após apresentarem acurácia acima de $90 \%$.

As seqüências de nucleotídeos foram alinhadas através do programa CLUSTAL X, versão 1.8 para Windows (Thompson et al. 1997). Como referência para o alinhamento foram utilizadas sequiências dos subtipos A, B, C, D e E do FIV disponíveis no GenBank (Fig.1).

Na determinação do modelo evolutivo mais adequado para substituição de nucleotídeos, utilizou-se o teste de razão de verossimilhança com o auxílio do programa Modeltest (Posada \& Crandall 1998), o qual indicou o método de Tamura-Nei (Tamura \& Nei 1993) com distribuição gama no valor de 0,26. Os valores de consistência foram obtidos através do teste de bootstrap, assumindo o valor de 1000 réplicas e com retenção de grupos com frequiência acima de $60 \%$. A árvore filogenética foi construída com o auxílio do programa MEGA (Molecular Evolutionary Genetics Analysis), versão 3.1 para Windows (Kumar et al. 2004).

\section{RESULTADOS E DISCUSSÃO}

As 23 sequiências analisadas neste trabalho foram alocadas dentro do subtipo B do FIV (Fig.1). Adicionalmente, pelo menos três subgrupos (1, 2 e 3 ) foram consistentemente identificados dentro do subtipo $\mathrm{B}$, como indicado pelos valores de bootstrap. O subgrupo 1 foi formado por 15 cepas paulistas, subgrupo 2 por apenas duas cepas estudadas e o subgrupo 3 foi formado apenas por uma das amostras (MogidasCruzes283) e por cinco cepas do FIV oriundas do Japão. As outras cinco cepas foram também alocadas no subtipo $\mathrm{B}$, porém não formaram subgrupos.

A identificação de um único subtipo nas amostras estuda- 


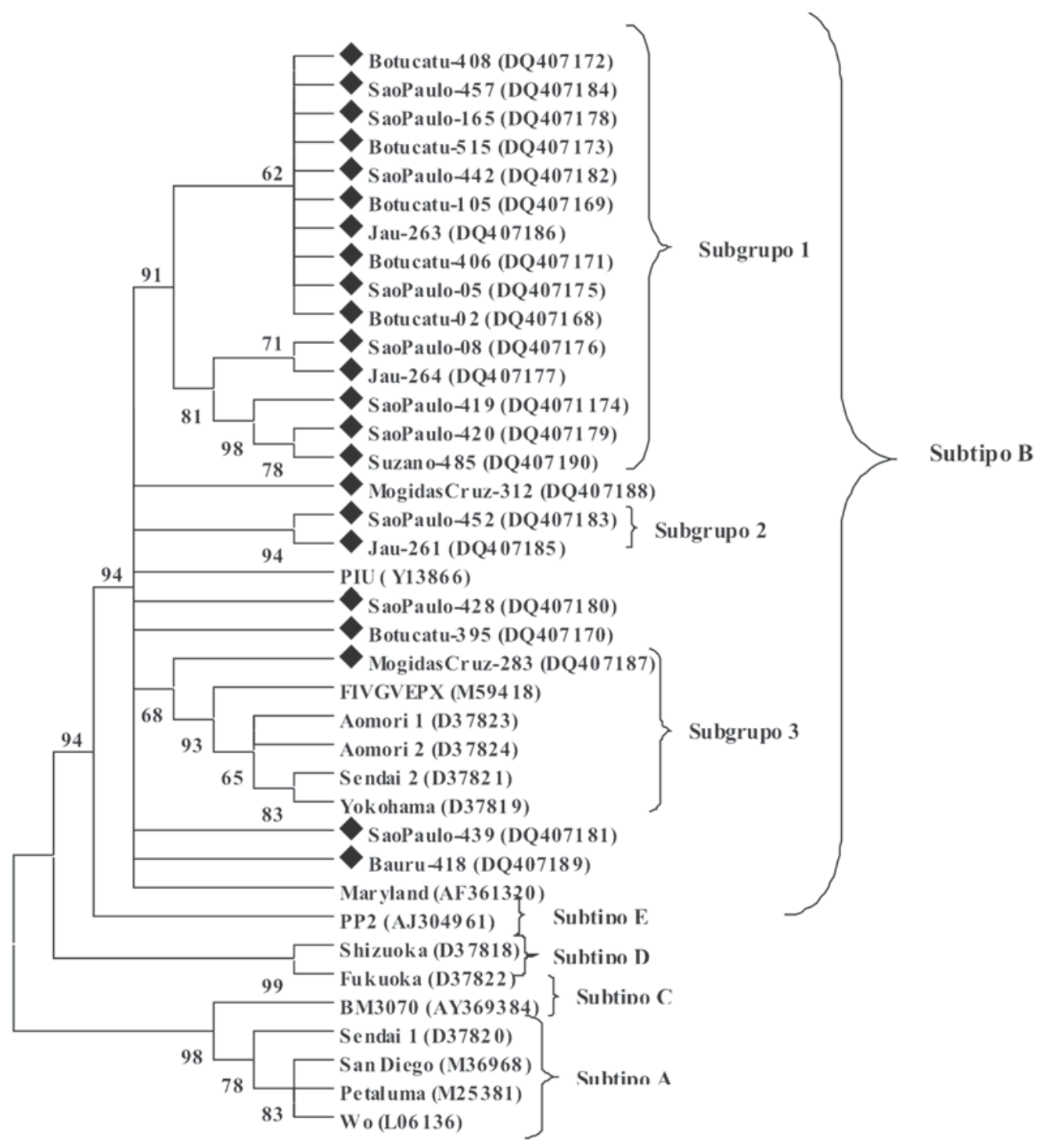

Fig.1. Árvore filogenética construída pelo método de Neighbor-Joining obtida através da análise de 23 sequiências de nucleotídeos do gene gag do vírus da imunodeficiência felina do Estado de São Paulo, Brasil. Os números nos nós indicam os valores de bootstrap para 1000 réplicas e o símbolo $\mathrm{f} \&$ as amostras analisadas.

das foi similar ao resultado obtido em Minas Gerais (Caxito et al. 2006), o que caracteriza, até o presente momento, a ocorrência de um único subtipo do FIV na região sudeste do Brasil. Entretanto, em Portugal, um estudo prévio de filogenia também identificou somente a ocorrência do subtipo B (Duarte et al. 2002). Posteriormente, em outro estudo utilizando amostras de diferentes cidades de Portugal identificou-se também a presença do subtipo A e de um possível novo subtipo, denominado de F (Duarte \& Tavares 2005). Esses resultados encontrados em Portugal vêm acrescentar a importância dos trabalhos já realizados no Brasil e da necessidade de estudos com maior número de amostras para se conhecer o real cenário da diversidade genética do FIV no País. Vale ressaltar que o presente estudo, por ter utilizado somente amostras do Estado de São Paulo, pode não refletir as características genéticas da população felina brasileira.
Como observado neste trabalho, a existência de subgrupos dentro do subtipo B já havia sido descrita (Steinrigl \& Klein 2003, Weaver et al. 2004), inclusive no Brasil (Caxito et al. 2006). A maior implicação para a formação de subgrupos dentro de um subtipo consiste na dificuldade de se utilizar diagnóstico baseado em biologia molecular (Steinrigl \& Klein 2003). Assim, identificação dos subtipos (e possíveis subgrupos) é essencial para a escolha da melhor região de amplificação do genoma do FIV. Isso também pode trazer conseqüências para o desenvolvimento de vacinas, cujas cepas presentes podem não proteger os animais para subtipos heterólogos (Reggeti \& Bienzle 2004).

Infelizmente, não foi possível rastrear a origem geográfica dos animais estudados, principalmente porque a maioria vivia em associações protetoras dos animais e a origem exata dos mesmos era desconhecida. O conhecimento da origem dos 
animais poderia trazer uma contribuição importante para o rastreamento evolutivo das cepas do FIV estudadas. Entretanto, a formação de um subgrupo com a amostra MogidasCruzes283 e os isolados japoneses e o alto valor de bootstrap obtido sugerem um possível ancestral comum entre estes. Esse resultado havia sido observado também nas cepas estudadas em Minas Gerais, as quais, possivelmente, possuem um ancestral comum com cepas japonesas do FIV (Caxito et al 2006).

Em resumo, os resultados obtidos detectaram a ocorrência de um único subtipo do FIV no estado de São Paulo. Entretanto, deve-se ressaltar que esses resultados são restritos à população de gatos investigados. Trabalhos subseqüentes abrangendo um número maior de amostras são necessários para determinar a realidade atual acerca da ocorrência e distribuição dos subtipos do FIV no estado de São Paulo e, igualmente, do Brasil.

Agradecimentos.- A todos os médicos veterinários e, especialmente, ao Prof. Dr. Adriano Bonfim Carregaro pela ajuda na colheita dos espécimes. A CAPES pelo fornecimento da bolsa de fomento e a FAPESP pelo auxílio à pesquisa (Proc.02/02649-0).

\section{REFERÊNCIAS}

Arjona A., Barquero N., Domenech A., Tejerizo G., Collado V.M., Toural C., Martin D. \& Gomez-Lucia E. 2007. Evaluation of a novel nested PCR for the routine diagnosis of feline leukemia virus (FeLV) and feline immunodeficiency virus (FIV). J. Feline Med. Surg. 9:14-22.

Bachmann M.H., Mathiason-Rubard C., Learn G.H., Rodrigo A.G., Sodora D.L., Mazzetti P., Hoover E.A. \& Mullins J.I. 1997. Genetic diversity of feline immunodeficiency virus: dual infection, recombination, and distinct evolutionary rates among envelope sequence clades. J. Virol. 71:4241-4253.

Bandechi P., Dell'Omodarme M. \& Magi M. 2006. Feline leukaemia virus (FeLV) and feline immunodeficiency virus infections in cats in the Pisa district of Tuscany, and attempts to control FeLV infection in a colony of domestic cats by vaccination. Vet. Rec. 158:555-557.

Caldas A.P.F., Leal E.S., Silva E.F.A. \& Ravazzolo A.P. 2000. Detecção do provírus da imunodeficiência felina em gatos domésticos pela técnica de reação em cadeia da polimerase. Pesq. Vet. Bras. 20:20-25.

Caxito F.A., Coelho F.M., Oliveira M.E. \& Resende M. 2006. Phylogenetic analysis of feline immunodeficiency virus strain from State of Minas Gerais, Brazil. Arq. Bras. Med. Vet. Zootec. 58:1222-1225.

Clavel F., Guyader M., Guetard D., Salle M., Montagnier L. \& Alizon M. 1986. Molecular cloning and polymorphism of the human immune deficiency virus type 2. Nature 324:691-695.

Duarte A., Marques M.I., Tavares L. \& Fevereiro M. 2002. Phylogenetic analysis of five Portuguese strains of FIV. Arch. Virol. 147:1061-1070.

Duarte A. \& Tavares L. 2005. Phylogenetic analysis of Portuguese feline immunodeficiency virus sequences reveals high genetic diversity. Vet. Microbiol. 114:25-33.

Ewing B., Hillier L., Wendl M.C. \& Green P. 1998. Base-calling of automated sequencer traces using Phred. I. Accuracy assessment. Genome Res. 8:175185.

Fisher A.G., Ensoli B., Looney D., Rose A., Gallo R.C., Saag M.S., Shaw G.M., Hahn B.H. \& Wong-Staal F. 1988. Biologically diverse molecular variants within a single HIV-1 isolate. Nature 334: 444-447.

Greene W.K., Meers J., Chadwick B., Carnegie P.R. \& Robinson W.F. 1993. Nucleotide sequences of Australian isolates of the feline immunodeficiency virus: comparison with other feline lentiviruses. Arch. Virol. 132:369-379.

Hohdatsu T., Motokawa K., Usami M., Amioka M., Okada S. \& Koyama H. 1998. Genetic subtyping and epidemiological study of feline immunodeficiency virus by nested polymerase chain reaction-restriction fragment lenght polymorphism analysis of the gag gene. J. Virol. Methods 70:107-111.

Inada G., Miyazawa T., Inoshima Y., Kohmoto M., Ikeda Y., Liu C.H., Lin J.A., Kou T.F. \& Mikami T. 1997. Phylogenetic analysis of feline immunodeficiency virus isolated from cats in Taiwan. Arch. Virol. 142:1459-1467.

Kakinuma S., Motokawa K., Hohdatsu T., Yamamoto J.K., Koyama H. \& Hashimoto H. 1995. Nucleotide sequence of feline immunodeficiency virus: classification of Japanese isolates into two subtypes which are distinct from non-japanese subtypes. J. Virol. 69:3639-3646.

Kumar S., Tamura K. \& Nei M. 2004. MEGA3: Integrated software for molecular evolutionary genetics analysis and sequence alignment. Brief. Bioinform. 5:150-163.

Lara V.M., Taniwaki S.A. \& Araújo Jr. J.P. 2001. Occurrence of feline immunodeficiency virus in domestic cats naturally infected. Virus Rev. Res. 06:173.

Levy J.K., Scott H.M., Lachtara J.L. \& Crawford P.C. 2006. Seroprevalence of feline leukemia virus and feline immunodeficiency virus infection among cats in North America and risk factors for seropositivity. J. Am. Vet. Med. Assoc. 228:371-376.

Luria B.J., Levy J.K., Lappin M.R., Breitschwerdt E.B., Legendre A.M., Hernandez J.A., Gorman S.P. \& Lee I.T. 2004. Pevalence of infectious diseases in feral cats in northern Florida. J. Fel. Med. Surg. 6:287-296.

Nishimura Y., Goto Y., Pang H., Endo Y., Mizuno T., Momoi Y., Watari T., Tsujimoto H. \& Hasegawa A. 1998. Genetic heterogeneity of env gene of feline immunodeficiency virus obtained from multiple districts in Japan. Virus Res. 57:101-112.

Pecoraro M.R., Tomonaga K., Miyazawa T., Kawaguchi Y., Sugita S., Tohya Y., Kai C., Etcheverrigaray M.E. \& Mikami T. 1996. Genetic diversity of Argentina isolates of feline immunodeficiency virus. J. Gen. Virol. 77:2031-2035.

Pedersen N.C., Ho E.W., Brown M.L. \& Yamamoto J.K. 1987. Isolation of a Tlymphotropic virus from domestic cats with an immunodeficiency-like syndrome. Science 235:790-793.

Pistello M., Cammarota G., Nicoletti E., Matteucci D., Curcio M., Mauro D.D. \& Bendinelli M. 1997. Analysis of the genetic diversity and phylogenetic relationship of the Italian isolates of feline immunodeficiency virus indicates a high prevalence and heterogeneity of subtipo B. J. Gen. Virol. 78:2247-2257.

Posada D. \& Crandall K.A. 1998. MODELTEST: testing the model of DNA substitution. Bioinform. Appl. 14:817-818.

Reche Jr A., Hagiwara M.K. \& Lucas S.R.R. 1997. Clinical study of acquired immunodeficiency syndrome in domestic cats in São Paulo. Braz. J. Vet. Res. Anim. Sci. 34:152-155.

Reggeti F. \& Bienzle D. 2004. Feline immunodeficiency virus subtypes A, B and $\mathrm{C}$ and intersubtype recombinants in Ontario, Canada. J. Gen. Virol. 85:1843-1852.

Sodora D.L., Shaper E.G., Kitchell B.E., Dow S.W., Hoover E.A. \& Mullins J.I. 1994. Identification of three feline immunodeficiency virus (FIV) env gene subtypes and comparison of the FIV and human immunodeficiency virus type 1 evolutionary patterns. J. Virol. 68:2230-2238.

Souza H.J.M., Teixeira C.H.R. \& Graça R.F.S. 2002. Estudo epidemiológico de infecções pelo vírus da leucemia e/ou imunodeficiência felina, em gatos domésticos do Município do Rio de Janeiro. Clin. Vet. 36:14-21.

Steinrigl A. \& Klein D. 2003. Phylogenetic analysis of feline immunodeficiency virus in Central Europe: a prerequisite for vaccination and molecular diagnostics. J. Gen. Virol. 84:1301-1307.

Tamura K. \& Nei M. 1993. Estimation of the number of nucleotide substitution in the control region of mitochondrial DNA in humans and chimpanzees. Mol. Biol. Evol. 10:512-526.

Thompson J.D., Gibson T.J., Plewniak F., Jeanmougin F. \& Higgins D.G. 1997. The CLUSTAL_X windows interface: flexible strategies for multiple sequence alignment aided by quality analysis tools. Nucleic Acids Res. 24:4876-4882.

Weaver E.A., Collisson E.W., Slater M. \& Zhu G. 2004. Phylogenetic analysis of Texas isolates indicate an evolving sbtype of the clade B Feline Immunodeficincy Viruses. J. Virol. 78:2158-2163. 